\title{
Adubação orgânica e cobertura do solo no crescimento e produção de camapu
}

\section{Nei Peixoto ${ }^{1}$; Fabrício de C Peixoto² ${ }^{2}$ Úrsula L Vaz ${ }^{1}$; Sarah Cristine M Neri ${ }^{1}$; Juliana G Monteiro ${ }^{1}$}

${ }^{1}$ UEG, Rod. GO 330, km 241, Anel Viário, 75780-000 Ipameri-GO; ${ }^{2}$ UNESP, Via de Acesso Paulo Donato Castelanni, km 05, 14870-000 Jaboticabal-SP; nei.p@terra.com.br; fcpeixoto81@yahoo.com.br; ursula_lv22@hotmail.com; sarah.crist@hotmail.com; geseira@bol. com.br;

\section{RESUMO}

Foi implantado um experimento na UEG, em Ipameri-GO, visando estudar os efeitos da adubação orgânica residual e cobertura do solo no desenvolvimento e produção de camapu. A semeadura foi feita em bandejas de poliestireno expandido em 01/10/2007 e as mudas foram mantidas em telado com $50 \%$ de sombreamento, até a data de plantio no campo, em 27/11/2007, em sucessão ao plantio de tomate. O delineamento experimental utilizado foi em blocos casualizados, com três repetições, com parcelas subdivididas, tendo na parcela duas doses de esterco de curral curtido (30 e $60 \mathrm{t} \mathrm{ha}^{-1}$ ) e nas sub-parcelas três tipos de cobertura do solo (plástico preto, capim braquiária e sem cobertura). Cada sub-parcela foi constituída por 20 plantas, dispostas no espaçamento de $1,00 \times 0,30 \mathrm{~m}$. A cobertura plástica favoreceu tanto o crescimento das plantas, quanto a produtividade. $\mathrm{O}$ efeito do esterco de curral foi pouco evidenciado. As curvas de crescimento em altura seguiram modelos cúbicos de regressão.

Palavras-chave: Physalis pubescens, altura da planta, produtividade.

\begin{abstract}
Plant growth and production of husk tomato depending on organic fertilization and mulching

A field trial was carried out in Ipameri, State of Goias, Brazil, to study the effect of residual cattle manure and soil covering on plant growth and yield of husk tomato. Sowing was carried out in October 01, and seedlings were kept under greenhouse with 50\% of shadow, until planting in the field in November 11, 2007, after tomato crop. The experimental design was randomized block with split-plot scheme with three replications. Cattle manure treatments were applied to the plots (30 and $60 \mathrm{tha}^{-1}$ ) and soil covering to subplots (black plastic, Brachiaria grass and without covering). The sub plot consisted of 20 plants arranged at space of $1.00 \times 0.30 \mathrm{~m}$. Black plastic covering favored plant growth and yield. Minor effects were obtained with residual cattle manure. Plant height followed cubic regression model.
\end{abstract}

Keywords: Physalis pubescens, plant height, yield.

(Recebido para publicação em 11 de fevereiro de 2009; aceito em 31 de agosto de 2010) (Received on February 11, 2009; accepted on August 31, 2010)

$\mathrm{H}_{\mathrm{s}}^{a}$ á controvérsias quanto ao centro de origem do camapu (Physalis sp.), visto que espécies do gênero são encontradas nos diversos continentes, mas a origem americana é considerada como mais provável (CIAT, 2004; Rufato et al., 2008).

No Brasil diversas espécies do gênero, entre elas a $P$. pubescens, ocorrem espontaneamente, sendo seus frutos bem doces consumidos in natura ou na forma de sucos por habitantes do meio rural, apresentando grande potencial para introdução ao cultivo (Menichelli, 2004).

As espécies Physalis angulata e Physalis peruviana são consideradas iguaria em restaurantes sofisticados, sendo cultivadas na América Latina, principalmente na Colômbia, onde ocupa a segunda posição na pauta de exportação do país, segundo Rufato et al. (2008). Nesse país tais espécies têm recebido atenção especial, tanto pelo segmento produtivo, quanto pela pesquisa (Colômbia, 2008; González et al., 2008).

Algumas espécies do gênero Physalis são nativas do Brasil, mas, por serem tratadas como planta daninha (Erasmo et al., 2004) e, como hospedeiras de doenças (Santos et al., 2004; Silva et al., 2006), têm perdido variabilidade ao longo do tempo.

$\mathrm{O}$ extrato da planta de camapu tem efeito moluscicida (Santos et al., 2003) e medicinal (Silva \& Agra, 2005; Lopes et al., 2006; Rufato et al., 2008).

A partir de estudos desenvolvidos na Estação Experimental Santa Luzia, no estado de São Paulo, espécies do gênero vêm despertando o interesse de produtores, sendo que hoje os maiores polos de expansão da cultura se encontram nos estados de Santa Catarina e Rio Grande do Sul (Rufato et al., 2008).

O objetivo desse trabalho foi estudar o crescimento e a produção de camapu
(Physalis pubescens), em resposta à adubação orgânica e da cobertura do solo.

\section{MATERIAL E MÉTODOS}

Foi implantado um experimento na UEG em Ipameri-GO, visando estudar o efeito residual de adubação orgânica e cobertura do solo no desenvolvimento e produção de camapu, em sucessão ao tomate cereja. A semeadura, utilizandose sementes de plantas nativas locais, foi feita em bandejas de poliestireno expandido em 01/10/2007, mantida sob telado, com $50 \%$ de sombreamento, até a data de plantio no campo, o que ocorreu aos 57 dias após a semeadura, nas mesmas unidades experimentais utilizada na cultura anterior.

Antes do plantio do tomate o solo apresentava as características químicas: $\mathrm{pH} 5,6 \mathrm{em} \mathrm{CaCl}_{2}, \mathrm{~V} 33 \%$, MO $23 \mathrm{~g} \mathrm{dm}^{3}$, P $30 \mathrm{mg} \mathrm{dm}^{-3}$ e os seguintes valores em mmolc dm ${ }^{-3}$ : Al 1,6; H+Al 47; K 1,7; 
Ca 16; Mg 5; saturação de bases 23 e CTC 70 .

O delineamento experimental utilizado foi em blocos casualizados, com parcelas subdivididas, tendo na parcela os resíduos de duas doses de esterco de curral curtido $\left(\mathrm{P} 1=30\right.$ e $\left.\mathrm{P} 2=60 \mathrm{tha}^{-1}\right)$ aplicado para a cultura do tomate e nas sub-parcelas três tipos de cobertura do solo $(\mathrm{S} 1=$ sem cobertura, $\mathrm{S} 2=$ capim braquiária e $\mathrm{S} 3=$ plástico preto), também remanescentes da cultura anterior e três repetições, sendo cada sub-parcela constituída por 20 plantas, dispostas no espaçamento de $1,00 \mathrm{~m} \times 0,30 \mathrm{~m}$, sendo consideradas úteis as 16 plantas centrais.

$\mathrm{Na}$ cultura anterior, de tomate tipo cereja, utilizou-se, como adubação química no plantio, comum aos tratamentos, $200 \mathrm{~kg} \mathrm{ha}^{-1}$ de N, $800 \mathrm{~kg} \mathrm{ha}^{-1}$ de $\mathrm{P}_{2} \mathrm{O}_{5}$ e $700 \mathrm{~kg} \mathrm{ha}^{-1}$ de $\mathrm{K}_{2} \mathrm{O}$ e, em cobertura, $300 \mathrm{~kg} \mathrm{ha}^{-1}$ do formulado 20-00-20, aos 30 dias após plantio. Não foi feita adubação química adicional para a cultura de camapu, além da residual do plantio anterior. Foi utilizada irrigação, por gotejamento, de forma a atender às necessidades hídricas da cultura e capinas, quando necessárias. Não foram feitas pulverizações com defensivos, visando avaliar a rusticidade da espécie.

Foram obtidos dados de altura das plantas, a partir de 21 dias após o plantio definitivo a cada 14 dias, até início de colheita, e de produção em número e massa de frutos por parcela, sendo as colheitas realizadas duas vezes por semana, a partir de 12/02/2008, durante um mês, colhendo-se frutos com sinais de maturação na planta, evidenciado pela cor amarelada e recém caídos das plantas. Estes foram submetidos às análises de variância, utilizando o aplicativo ESTAT. As médias de doses de esterco e tipo de cobertura foram comparadas pelo teste de Tukey, com 5\% de probabilidade de erro e as curvas de crescimento em altura em função do tempo definidas por análise de regressão.

\section{RESULTADOS E DISCUSSÃO}

Foi observada elevada incidência de ácaro branco na cultura o que provocou, no início de florescimento, perdas de

Tabela 1. Altura das plantas, (cm) aos 21, 35, 49, 63 e 79 dias após transplante (DAT), para seis combinações de adubação residual com esterco de curral e cobertura do solo (plant height (cm) at 21, 35, 49, 63 and 79 days after transplanting (DAT), for six combinations of residual fertilization with cattle manure and mulching). Ipameri, UEG, 2007-2008.

\begin{tabular}{lcllcc}
\hline Tratamento & 21 DAT & 35 DAT & 49 DAT & 63 DAT & 79 DAT \\
\hline P1S1 & $5,47 \mathrm{a}$ & $36,84 \mathrm{a}$ & $81,22 \mathrm{a}$ & $105,82 \mathrm{a}$ & $120,00 \mathrm{a}$ \\
P1S2 & $6,87 \mathrm{a}$ & $22,89 \mathrm{ab}$ & $61,38 \mathrm{bc}$ & $93,98 \mathrm{ab}$ & $110,00 \mathrm{ab}$ \\
P1S3 & $5,31 \mathrm{a}$ & $21,90 \mathrm{~b}$ & $61,66 \mathrm{bc}$ & $87,13 \mathrm{~b}$ & $96,33 \mathrm{~b}$ \\
P2S1 & $5,54 \mathrm{a}$ & $23,94 \mathrm{ab}$ & $63,36 \mathrm{bc}$ & $99,89 \mathrm{ab}$ & $116,67 \mathrm{a}$ \\
P2S2 & $5,86 \mathrm{a}$ & $20,26 \mathrm{~b}$ & $56,64 \mathrm{c}$ & $88,54 \mathrm{~b}$ & $106,00 \mathrm{ab}$ \\
P2S3 & $6,80 \mathrm{a}$ & $26,76 \mathrm{ab}$ & $74,02 \mathrm{ab}$ & $97,34 \mathrm{ab}$ & $117,00 \mathrm{a}$ \\
\hline
\end{tabular}

$\mathrm{CV}(\%)$ 15,32

Médias seguidas da mesma letra, na coluna, não diferem entre si pelo teste de Tukey, ao nível de $5 \%$ de probabilidade (means followed by the same letter in column do not differ from each other, by the Tukey's test, $\mathrm{p}<0.05$ ); P1 e P2= doses de 30 e $60 \mathrm{~m}^{3} \mathrm{ha}^{-1}$ de esterco de curral, respectivamente; S1, S2 e S3= cobertura do solo com plástico preto, capim brachiaria e sem cobertura, respectivamente $\left(\mathrm{P} 1\right.$ and $\mathrm{P} 2=$ dose of 30 and $60 \mathrm{~m}^{3} \mathrm{ha}^{-1}$ of cattle manure, respectively; $\mathrm{S} 1, \mathrm{~S} 2$ and $\mathrm{S} 3=$ soil covering with black plastic, brachiaria grass and without covering, respectively).

Tabela 2. Altura das plantas, $(\mathrm{cm})$, em cada dose de esterco e produtividade, em função do tipo de cobertura do solo (plant height $(\mathrm{cm})$ in each dose of cattle manure, and yield, in response of mulching). Ipameri, UEG, 2007-2008.

\begin{tabular}{|c|c|c|c|c|c|}
\hline \multirow[b]{2}{*}{ Cobertura do solo } & \multicolumn{2}{|c|}{ Altura aos 35 DAT } & \multicolumn{2}{|c|}{ Altura aos 49 DAT } & \multirow{2}{*}{$\begin{array}{c}\text { Produti- } \\
\text { vidade } \\
\left(\mathrm{t} \mathrm{ha}^{-1}\right)\end{array}$} \\
\hline & $\begin{array}{c}\text { Esterco } \\
\left(30 \mathrm{~m}^{3} \mathrm{ha}^{-1}\right)\end{array}$ & $\begin{array}{c}\text { Esterco } \\
\left(60 \mathrm{~m}^{3} \mathrm{ha}^{-1}\right)\end{array}$ & $\begin{array}{c}\text { Esterco } \\
\left(30 \mathrm{~m}^{3} \mathrm{ha}^{-1}\right. \\
\end{array}$ & $\begin{array}{c}\text { Esterco } \\
\left(60 \mathrm{~m}^{3} \mathrm{ha}^{-1}\right)\end{array}$ & \\
\hline Plástico preto (S1) & $36,84 \mathrm{a}$ & $23,94 \mathrm{ab}$ & $81,22 \mathrm{a}$ & $63,36 \mathrm{ab}$ & $13,00 \mathrm{a}$ \\
\hline Capim (S2) & $22,89 \mathrm{~b}$ & $20,26 \mathrm{~b}$ & $61,38 \mathrm{~b}$ & $56,64 \mathrm{~b}$ & $10,31 \mathrm{~b}$ \\
\hline Sem cobertura $(\mathrm{S} 3)$ & $21,90 \mathrm{~b}$ & $26,76 \mathrm{a}$ & $61,66 \mathrm{~b}$ & $74,02 \mathrm{a}$ & $10,12 \mathrm{~b}$ \\
\hline $\mathrm{CV}(\%)$ & \multicolumn{2}{|c|}{9,38} & \multicolumn{2}{|c|}{7,45} & 12,27 \\
\hline
\end{tabular}

Médias seguidas da mesma letra, na coluna, não diferem entre si pelo teste de Tukey, ao nível de $5 \%$ de probabilidade (means followed by the same letter in column do not differ from each other, by Tukey's test, $\mathrm{p}<0.05)$.

botões florais, não quantificadas, o que pode ter interferido no rendimento da cultura.

Foram significativos os efeitos de doses de esterco de curral e cobertura de solo e da interação entre tratamentos e épocas de medidas das plantas. $\mathrm{Na}$ primeira medida os tratamentos se igualaram estatisticamente. Nas demais o tratamento P1S1 (30 $\mathrm{m}^{3} \mathrm{ha}^{-1}$ de esterco e cobertura do solo com plástico preto) apresentou as plantas mais altas em todas as observações, mas igualando-se, sempre à P2S3 $\left(60 \mathrm{~m}^{3} \mathrm{ha}^{-1}\right.$ e solo sem cobertura), mostrando haver confundimento entre os dois fatores (Tabela 1).

Quando isolados os efeitos dos fatores, observou-se não haver diferenças significativas para altura de plantas em relação às doses de esterco para a média das medidas, mas na segunda e terceira medidas, os tratamentos com $30 \mathrm{~m}^{3} \mathrm{ha}^{-1}$ de esterco de curral e cobertura plástica proporcionaram maiores alturas.

O número médio de frutos por planta foi de 377 , sem haver diferenças significativas entre os tratamentos. Para produtividade, foram significativos apenas os efeitos da cobertura do solo, sendo que a cobertura com plástico preto foi a mais favorável, quanto à média das doses de esterco (Tabela 2). Não foram encontradas na literatura consultada informações sobre a produtividade de Physalis pubescens, nem sobre o seu cultivo e comercialização, ao contrário de outras espécies do gênero.

As curvas de crescimento de todos os tratamentos seguiram modelos cúbicos, mostrando crescimento lento e contínuo, no período inicial, mais lento após o início de frutificação e mais acentuado nas 


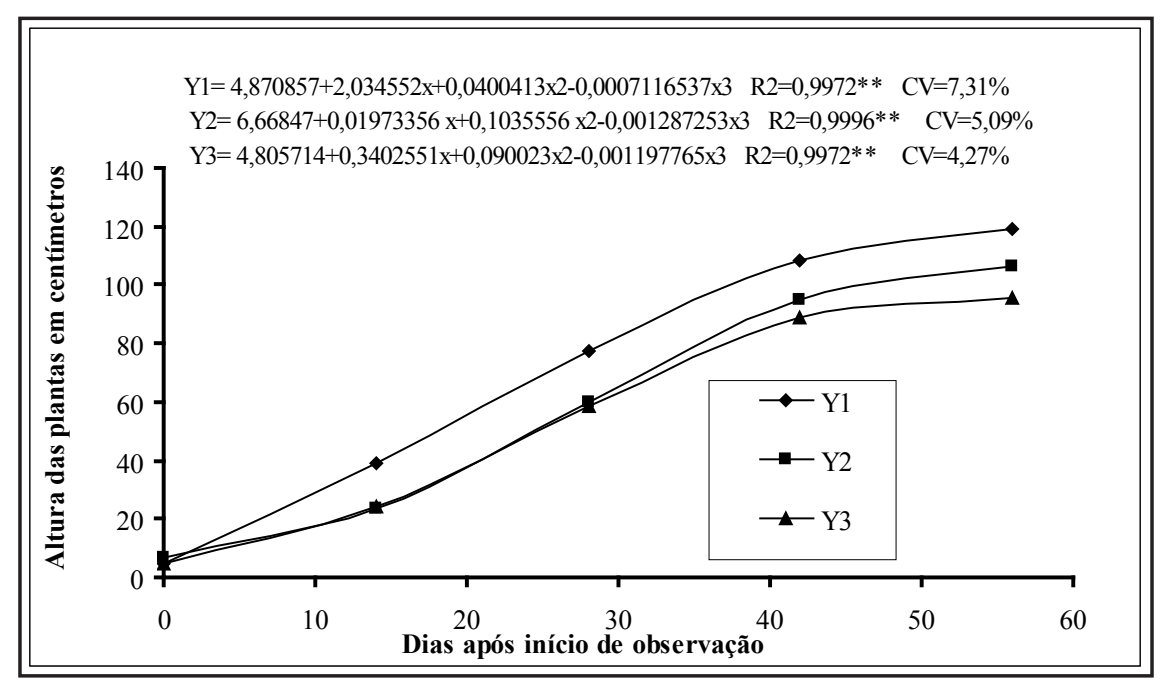

Figura 1. Altura média das plantas de camapu em função da idade, na dose de $30 \mathrm{~m}^{3} \mathrm{ha}^{-1}$ de esterco de curral com cobertura plástica (Y1), capim (Y2) e sem cobertura (Y3) do solo (husk tomato plant average height depending on plant age in $30 \mathrm{~m}^{3} \mathrm{ha}^{-1}$ of cattle manure and soil mulch with plastic (Y1), grass (Y2) and without mulch (Y3)). Ipameri, UEG, 2007-2008.

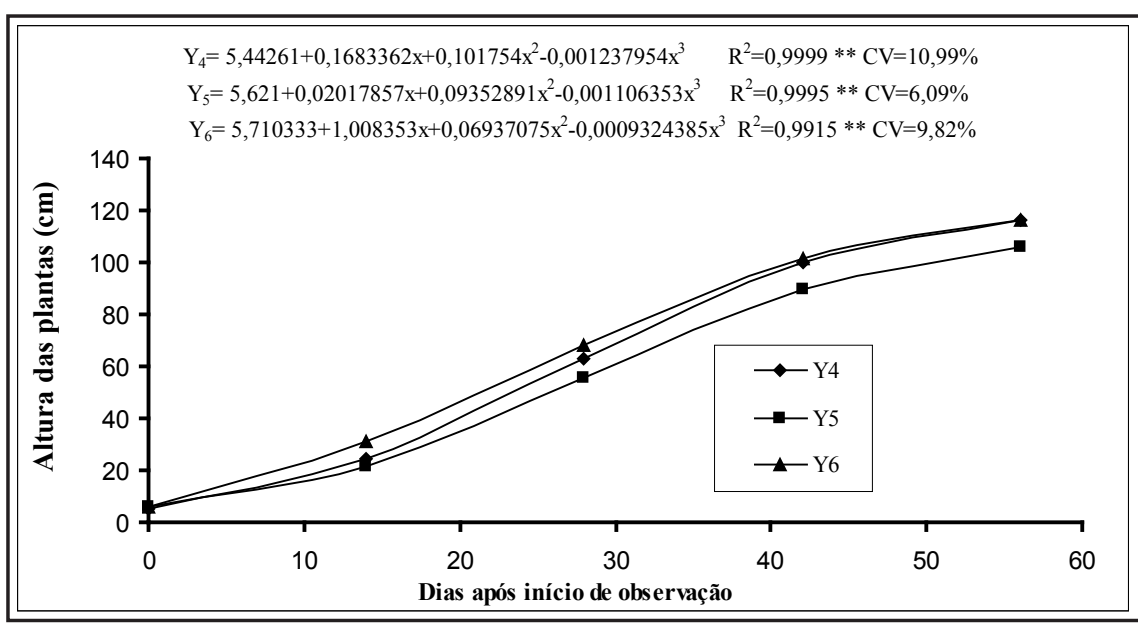

Figura 2. Altura média de plantas de camapu em função da idade, na dose de $60 \mathrm{~m}^{3} \mathrm{ha}^{-1} \mathrm{de}$ esterco de curral com cobertura plástica (Y4), capim (Y5) e sem cobertura (Y6) do solo (husk tomato plant average height depending on plant age in $60 \mathrm{~m}^{3} \mathrm{ha}^{-1}$ of cattle manure and soil mulch with plastic (Y4), grass (Y5) and without mulch (Y6)). Ipameri, UEG, 2007-2008.

observações finais (Figuras 1, 2).

Com base nesses resultados concluise que Physalis pubescens pode ser produzido em plantios sucessivos com outras hortaliças, aproveitando-se o efeito residual de fertilizantes. Entretanto, apresenta pragas que requerem controle para bom desempenho da cultura. Ao

\section{REFERÊNCIAS}

CIAT-Centro Internacional de Agricultura Tropical. 2004, 15 de setembro. Fruit from America. Disponível em: http://www.ciat.cgiar.org/ ipgri/fruits_from_americas/frutales/Ficha $\% 20$ Physalis\% $\%$ pubescens.htm. Acessado em 14 de setembro de 2004.

COLOMBIA - República de Colombia, Embajada de Colombia em Portugal. 2008, 7 de fevereiro. Physalis. Disponível em: http://www. embajadadecolombiaenportugal.com/physalis. $\mathrm{htm}$. Acessado em 10 de outubro de 2008.

ERASMO EAL; PINHEIRO LLA; COSTA NV. 2004. Levantamento fitossociológico das comunidades de plantas infestantes em áreas de arroz irrigado cultivado sob diferentes sistemas de manejo. Viçosa: Planta Daninha 22: 195-201.

GONZÁLEZ OT; TORRES JMC; CANO CIM; ARIAS ML; ARBOLEDA AAN. 2008. Caracterización morfológica de cuarenta y seis acessiones de uchuva (Physalis peruviana $\mathrm{L}$.), in Antioquia (Colombia). Revista Brasileira de Fruticultura 30: 708-715.

LOPES DCDXP; FREITAS ZMF; SANTOS E.P; TOMASSINI TCB 2006. Atividades antimicrobianas e fitotóxica de extratos de frutos e raízes de Physalis angulata L. Revista Brasileira de Farmacognosia 16: 206-210.

MENICHELLI C. 2004. Beleza escondida. Rio de Janeiro: Revista Época, Edição 135, jan. 2004. Disponivel em http://www2.uol.com.br/gula/ reportagens $/ 135$ physalis.shtml. Acessado em 14 de setembro de 2004.

RUFATO L; RUFATO AR; SCHLEMPER C; LIMA CSM; KRETZCHMAR AA. 2008. Aspectos técnicos da cultura da Physalis. Pelotas: UFPEL, 101p.

SANTOS CDG; D'ÁVILA AC; INONE-AGATA AK; REZENDE RO 2004. Espécies vegetais hospedeiras de begomovirus isolados de tomateiro em Goiás e Distrito Federal. Fitopatologia Brasileira 29: 450-455.

SANTOS JAA; TOMASSINI TCB; XAVIER DCD; RIBEIRO IM; SILVA MTG; MORAIS FILHO ZB. 2003. Molluscicidal activity of Physalis angulata L. extracts and fractions on Biomphalaria tenagophila under laboratory condictions. Memórias do Instituto Oswaldo Cruz 98: 425-428.

SILVA KN; AGRA MF. 2005. Estudo etnobotânico comparativo entre Nicandra physalodes e Physalis angulata (Solanaceae). Revista Brasileira de Farmacognosia 15: 344-351.

SILVA MCL; SANTOS CDG; SANTOS AA; LIMA JAA. 2006. Vírus da mancha amarela da gravioleira: transmissão, hospedeiros, purificação e produção de anti-soro. Ciência Agronômica 37: 160-165. 